\title{
Prenatal diagnosis of atrial isomerism in the Korean population
}

\author{
Mi-Young Lee ${ }^{1}$, Hye-Sung Won', Jae-Yoon Shim¹, Pil-Ryang Lee ${ }^{1}$, Byong Sop Lee², Ellen Ai-Rhan Kim², \\ Young-Hwue Kim³, Jeong-Jun Park ${ }^{4}$, Tae-Jin Yun ${ }^{4}$, Ahm Kim \\ ${ }^{1}$ Department of Obstetrics and Gynecology, ${ }^{2}$ Division of Neonatology, Department of Pediatrics, ${ }^{3}$ Department of Pediatric Cardiology, ${ }^{4}$ Division of \\ Pediatric Cardiac Surgery, University of Ulsan College of Medicine, Asan Medical Center, Seoul, Korea
}

\section{Objective}

To report our experiences in the prenatal diagnosis of atrial isomerism and postnatal outcomes.

\section{Methods}

A total of 80 fetuses prenatally diagnosed with atrial isomerism were retrospectively analyzed between 1999 and 2011 at a single institution.

\section{Results}

Of 43 fetuses with prenatally diagnosed right atrial isomerism (RAI), 40 cases were analyzed. The diagnostic accuracy was $93 \%$. The main intracardiac anomalies in RAI were atrioventricular septal defect (AVSD), abnormal pulmonary venous connection, bilateral superior vena cava (BSVC), and pulmonary atresia. Among 28 live births, three infants were lost to follow up, and the overall survival rate was $60 \%$. Of 37 fetuses with prenatally diagnosed left atrial isomerism (LAI), 35 were evaluated. The diagnostic accuracy was $97 \%$. The main intracardiac anomalies in LAI were ventricular septal defect, BSVC, AVSD, double outlet right ventricle, and bradyarrhythmia. Among seven patients with bradyarrhythmia, only one showed a complete atrioventricular block. All fetuses had an interrupted inferior vena cava with azygous continuation. The overall survival rate was $90 \%$.

\section{Conclusion}

Our study confirms the previous findings of fetal atrial isomerism. We also demonstrates a much lower prevalence of AVSD and complete heart block in LAI and a better survival rate in RAl. Although the postnatal outcomes for RAI were worse than those for LAl, successful postnatal surgery with active management improved the survival rate.

Keywords: Echocardiography; Heart defects, congenital; Heterotaxy syndrome; Prenatal diagnosis

\section{Introduction}

Atrial isomerism is a disorder characterized by the symmetrical development of normally asymmetrical organ systems and is associated with complex cardiovascular abnormalities. There are two types of atrial isomerism based upon the morphology of the atrial appendages, right atrial isomerism (RAI) and left atrial isomerism (LAI). Several studies regarding the spectrum of cardiovascular and visceral abnormalities in fetal atrial isomerism have been reported [1-10]. Most of them were reported in Western countries and there are few studies
Received: 2013.10.2. Revised: 2013.11.11. Accepted: 2013.11.11.

Corresponding author: Hye-Sung Won

Department of Obstetrics and Gynecology, University of Ulsan College of Medicine, Asan Medical Center, 88 Olympic-ro 43-gil, Songpa-gu, Seoul 138-736, Korea

Tel: +82-2-3010-3644 Fax: +82-2-3010-6944

E-mail: hswon@amc.seoul.kr

Articles published in Obstet Gynecol Sci are open-access, distributed under the terms of the Creative Commons Attribution Non-Commercial License (http://creativecommons. org/licenses/by-nc/3.0/) which permits unrestricted non-commercial use, distribution, and reproduction in any medium, provided the original work is properly cited.

Copyright $\odot 2014$ Korean Society of Obstetrics and Gynecology 


\title{
Obstetrics \& Gynecology Science
}

\author{
Vol. 57, No. 3, 2014
}

regarding the characteristics of the atrial isomerism in Asian population.

Based on this observation, we decided to review data collected over a 12 year period in the Korean population, to report our experience in the prenatal diagnosis of atrial isomerism, including the accuracy of prenatal diagnosis, the spectrum of cardiac and other associated anomalies, and postnatal outcomes. We also compared the spectrum of atrial isomerism shown in our results with the findings of the previous reports.

\section{Materials and methods}

Following approval from the institutional review board, we retrospectively analyzed the 80 fetuses prenatally diagnosed with atrial isomerism between 1999 and 2011 at Asan Medical Center, Seoul, Korea. All cases were referred to our tertiary center because of suspected congenital heart disease or abnormal visceral situs. All patients received a detailed highresolution ultrasound, including color and pulsed wave Doppler. M-mode was used if the cardiac rhythm was abnormal. The examinations were performed using an Accuvix XG, XQ (Samsung Medison, Seoul, Korea) or Acuson Sequoia ultraound equipment (Acuson Inc., Mountain View, CA, USA).

RAI was suspected prenatally in the presence of characteristic cardiac anomalies, including atrioventricular septal defect, abnormal ventriculoarterial connection with pulmonary stenosis or atresia, anomalous pulmonary venous return, with at least one more common feature of RAl, such as juxtaposition of the aorta and inferior vena cava (IVC) on either side of the spine, or visceral heterotaxy. LAI was suspected in the presence of an interrupted IVC with azygos continuation and at least one more common feature of LAl, such as cardiac defects, heart block, or visceral heterotaxy. Spleen was also evaluated to aid the prenatal diagnosis of atrial isomerism (asplenia in RAI and polysplenia in LAI). The prenatal diagnosis was confirmed by various imaging techniques including echocardiography, cardiac computed tomography, abdominal ultrasound and surgical or autopsy records. The prenatal diagnosis and postnatal outcomes were assessed by reviewing medical records. Associated extracardiac anomalies including bowel malrotation, atresia, or hiatal hernia and chromosomal abnormalities were also evaluated.

The frequency of each cardiovascular anomaly was demon- strated as percentages. The gestational age at diagnosis and delivery were shown as a median with range.

\section{Results}

\section{Right atrial isomerism}

\section{1) Prenatal diagnosis}

Among 43 cases of prenatally diagnosed RAl, 12 terminated the pregnancy, one died in utero, and 30 survived. An autopsy was denied in three fetuses including one death in utero leaving a total of 40 fetuses for analysis. Three cases were postnatally or post-mortem diagnosed with complex heart disease other than RAl, thus making the diagnostic accuracy of RAI 93\% (37/40). Ten cases which were diagnosed with RAI postnatally rather than prenatally, were excluded from this study. The median gestational age at diagnosis was 28.4 weeks (range, 16.5-38.6 weeks). Out of 24 fetuses undergoing karyotyping, no abnormalities were found.

\section{2) Spectrum of cardiac and extracardiac anomalies} The spectrum of cardiovascular abnormalities is demonstrated in Table 1. The main intracardiac anomalies in RAI were atrioventricular septal defect, abnormal pulmonary venous connection, bilateral superior vena cava and pulmonary atresia. Among 25 cases of total anomalous pulmonary venous return, the most common type was supracardiac $(n=11,44 \%)$, followed by cardiac $(n=7,28 \%)$, infracardiac $(n=5,20 \%)$, and mixed type $(n=2,8 \%)$.

Table 2 summarizes the visceral abnormalities in RAI. The majority of RAI cases revealed juxtaposition of the descending aorta and IVC on the same side. Postnatal evaluation noted a normal spleen in two cases. One neonate had a horseshoe adrenal gland, which is a rare congenital condition caused by fusion of both adrenal glands.

\section{3) Postnatal outcomes}

Twenty-eight fetuses were born alive with a median gestational age of 38.3 weeks (range, 31.4-40.5 weeks). The outcomes of patients with RAl are shown in Fig. 1A. Among the live births, there were 10 deaths in neonatal, infant, or late periods. Five children are currently doing well without any severe complications after a successful Fontan type operation. Only one child is reporting a developmental delay due to se- 


\section{Obstetrics \& Gynecology Science}

Mi-Young Lee, et al. Prenatal diagnosis of atrial isomerism

Table 1. Spectrum of cardiovascular abnormalities in RAI and LAI

\begin{tabular}{|c|c|c|}
\hline Cardiac abnormality & RAI $(n=37)$ & LAI $(n=34)$ \\
\hline \multicolumn{3}{|l|}{ Cardiac position } \\
\hline Levocardia & $30(81)$ & $26(76)$ \\
\hline Dextrocardia/mesocardia & $7(19)$ & $8(25)$ \\
\hline \multicolumn{3}{|l|}{ Venous anomalies } \\
\hline Bilateral superior vena cava & $23(62)$ & $14(41)$ \\
\hline Total anomalous pulmonary venous return & $25(68)$ & $1(3)$ \\
\hline Partial anomalous pulmonary venous return & $1(3)$ & $2(6)$ \\
\hline \multicolumn{3}{|l|}{ Atria and ventricles } \\
\hline Atrioventricular septal defect & $32(86)$ & $9(26)$ \\
\hline \multicolumn{3}{|l|}{ Outflow tracts and great vessels } \\
\hline Double outlet right ventricle & $14(38)$ & $7(21)$ \\
\hline Transposition of great arteries & $5(14)$ & $2(6)$ \\
\hline Pulmonary stenosis & $14(38)$ & $4(12)$ \\
\hline Pulmonary atresia & $20(54)$ & $2(6)$ \\
\hline Major aortopulmonary collateral artery & $5(14)$ & $1(3)$ \\
\hline \multicolumn{3}{|l|}{ Arch anomalies } \\
\hline Coarctation of aorta & $1(3)$ & $3(9)$ \\
\hline Right aortic arch & $14(38)$ & $3(9)$ \\
\hline Arrhythmia & $0(0)$ & $7(21)$ \\
\hline
\end{tabular}

Values are presented as number (\%).

RAI, right atrial isomerism; LAI, left atrial isomerism.

quelae from Hemophilus influenza meningitis. The remaining nine children are waiting to undergo a single ventricle palliation. Consequently 15 patients are now alive at the median follow up of 35 months (range, 5-90 months) making the overall survival rate $60 \%(15 / 25)$.

\section{Left atrial isomerism}

\section{1) Prenatal diagnosis}

Out of 37 fetuses with prenatally diagnosed LAl, two had no postnatal confirmative diagnosis, leaving a total of 35 cases for analysis. In two cases, the pregnancy was terminated while the remaining 33 fetuses were born alive. There were no cases of fetal death. All but one case were postnatally or postmortem confirmed with LAl, thus the diagnostic accuracy was $97 \%$ (34/35). There were no cases of missed prenatal diagnosis during the study period.

The median gestational age at diagnosis was 25.3 weeks (range, 21-36.2 weeks). Among 26 fetuses undergoing karyotyping, one case of inv(9)(p12q13) was found, which is the most common pericentric inversion in the human karyotype and is considered to be a chromosomal variant in the normal population.

2) Spectrum of cardiac and extracardiac anomalies The most common intracardiac anomalies in LAI were ventricular septal defect $(n=17,50 \%)$, followed by bilateral superior vena cava, atrioventricular septal defect, double outlet right ventricle, and bradyarrhythmia (Table 1). The prevalence of complex cardiac malformation was lower than in RAI fetuses. Among seven patients with bradyarrhythmia, one showed a complete atrioventricular block, which eventually needed a pacemaker, and one revealed an intermittent heart block, which was normalized during the follow up period. The remaining patients showed sick sinus syndrome $(n=2)$, junctional rhythm $(n=1)$, non-conducted premature atrial contractions $(n=1)$ and sinus bradycardia $(n=1)$. Four neonates were confirmed as having a normal heart.

All fetuses had an interrupted IVC with azygos continuation (Table 2). One infant showed asplenia. Combined extracardiac anomalies are demonstrated in Table 2. 


\title{
Obstetrics \& Gynecology Science
}

\author{
Vol. 57, No. 3, 2014
}

Table 2. Spectrum of visceral abnormalities in RAI and LAI

\begin{tabular}{|c|c|c|}
\hline Visceral abnormality & RAI ( $n=37)$ & LAI $(n=34)$ \\
\hline Visceral heterotaxy & $36(97)$ & $20(59)$ \\
\hline Location of liver: midline/right/left & $35 / 0 / 1(97 / 0 / 3)$ & $14 / 0 / 6(70 / 0 / 30)$ \\
\hline Location of stomach: midline/right/left & $2 / 22 / 12(6 / 61 / 33)$ & $2 / 15 / 3(10 / 75 / 15)$ \\
\hline Asplenia & $35(95)$ & $1(3)$ \\
\hline Polysplesia & $0(0)$ & $24(71)$ \\
\hline Juxtaposition of the aorta and inferior vena cava & $30(81)$ & $0(0)$ \\
\hline Interrupted interior vena cava with azygos continuation & $0(0)$ & $34(100)$ \\
\hline \multicolumn{3}{|l|}{ Gastrointestinal abnormality } \\
\hline Intestinal malrotation & $8(22)$ & $7(21)$ \\
\hline Esophageal atresia & $0(0)$ & $1(3)$ \\
\hline Duodenal atresia & $3(8)$ & $1(3)$ \\
\hline Duodenal web & $0(0)$ & $1(3)$ \\
\hline Hiatal hernia & $3(8)$ & $1(3)$ \\
\hline Hirschsprung disease & $2(5)$ & $0(0)$ \\
\hline Biliary atresia & $0(0)$ & $1(3)$ \\
\hline Horseshoe adrenal gland & $1(3)$ & $0(0)$ \\
\hline Single umbilical artery & $1(3)$ & $2(6)$ \\
\hline Persistent right umbilical vein & $1(3)$ & $2(6)$ \\
\hline
\end{tabular}

Values are presented as number (\%).

RAI, right atrial isomerism; LAl, left atrial isomerism.
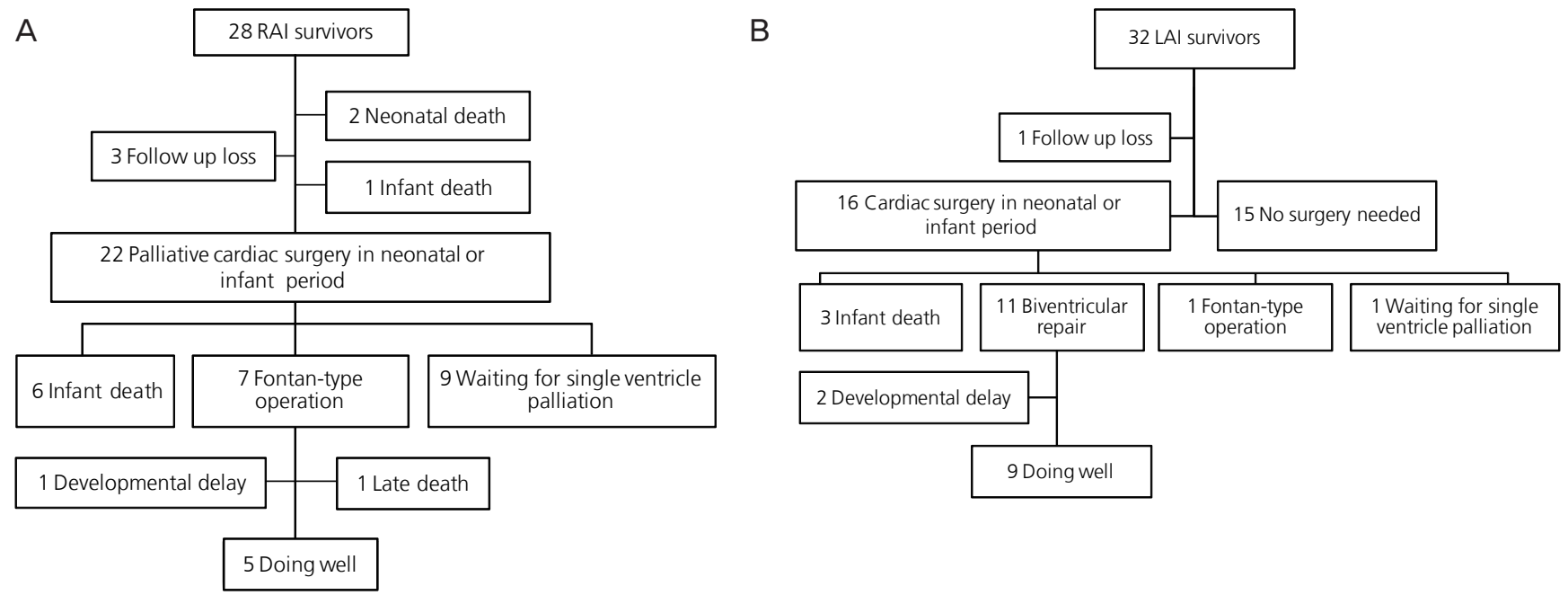

Fig. 1. Flow diagram showing the postnatal outcomes of fetuses diagnosed with right atrial isomerism (RAI, $A$ ) and left atrial isomerism (LAl, $B$ ).

\section{3) Postnatal outcomes}

The median gestational age at delivery of 32 fetuses was 38.4 weeks (range, 35.3-41.4 weeks). Fig. 1B shows the outcomes of patients with LAl. Fifteen neonates had no clinically significant cardiac defects or a normal heart. Among the 16 patients who underwent cardiac surgery, three infants died, two suffered developmental delays, and the remaining 11 children are doing well without severe complications at the median follow up of 18 months (range, 1-138 months). The overall survival rate was $90 \%(28 / 31)$. 


\section{Obstetrics \& Gynecology Science}

Mi-Young Lee, et al. Prenatal diagnosis of atrial isomerism

\section{Discussion}

Our study demonstrates the spectrum of cardiac and other associated anomalies in atrial isomerism with the large cohort, and confirms the previous findings of fetal atrial isomerism. Several studies carried out in Western countries showed a greater incidence of LAI compared to RAI. However among Asians, two studies showed that the incidence of RAI was higher than LAI $[4,9]$, and the authors suggested that there might be geographical heterogeneity in the expression of the two forms of atrial isomerism. Although this report includes the largest cohort of prenatally diagnosed RAI compared to previous studies [1-5,7-9], the predominence of RAI was not demonstrated and there was only small difference of the incidence between RAI and LAI in our study. To support the hypothesis that atrial isomerism may be racially related, further international studies are needed to confirm any genetic or environmental differences in predisposition according to ethnic groups.

The spectrum of cardiac and extracardiac abnormalities of atrial isomerism has been well documented. However the prevalence of each cardiac disease varies considerably in different studies. The typical intracardiac features of RAI are atrioventricular septal defect, total anomalous pulmonary venous return, ventriculoarterial discordance and pulmonary obstruction; these were similar to those observed in our study. LAI shows less severe cardiac defect, and is frequently associated with interrupted IVC, atrioventricular septal defect and complete heart block, or even a normal heart. As in previous studies, we showed that interrupted IVC with azygos vein continuation was the most common features in LAl; however we observed a much lower prevalence of atrioventricular septal defect (26\%) and complete heart block (3\%), and a greater survival rate $(90 \%)$ than in previous reports $[2,6$ $8,10]$. In addition, there were no cases of congestive heart failure, hydrops, or subsequent death in utero. A Taiwanese study revealed that among four fetuses with LAI, only one had a common atrioventricular canal and all suffered with bradycardia [4]. Another study from Singapore demonstrated that out of four cases of LAl, one had atrioventricular septal defect, and none had a heart block [9]. Because there is only a small number of prenatally diagnosed LAl cases reported in the Asian population, it is difficult to compare the spectrum of cardiac malformations between different ethnic groups. However we carefully suppose that racial differences could affect the disease spectrum.

Various gastrointestinal (GI) abnormalities may occur in cases of atrial isomerism [11,12]. These anomalies are caused by a failure in formation of asymmetry along the left right body axis, leading to defects in ligaments which fix the Gl tract [13]. Our results also demonstrated various combinations of Gl abnormalities. All anomalies in the Gl tract, except malrotation, were suspected during the prenatal period and were finally confirmed by postnatal evaluation. Intestinal malrotation is impossible to diagnose by prenatal ultrasound unless the signs of obstruction or volvulus are present. Midline liver, malposition of stomach, malrotation and malfixation are frequently found in both RAI and LAI [14]. Hiatal hernia is more commonly found in RAI, whereas biliary atresia is relatively common in LAI $[13,15]$. Hiatal hernia should be suspected when the hypoechogenic mass is present in the posterior mediastinum, just behind the heart, and the stomach is abnormally located, in a median position [16]. This condition was found in three RAI cases and one LAI case. Biliary atresia should be suspected when the gallbladder is non-visualized, but it is an unreliable marker [10]. This condition was found in one patient with LAl, who eventually underwent a liver transplantation. When atrial isomerism is prenatally diagnosed, the abdominal contents should also be carefully examined due to the high incidence of associated abdominal abnormalities.

There was one patient who pregnant the two siblings who were affected by RAl. The first pregnancy was terminated and RAI was confirmed by autopsy. She then delivered a normal full-term baby followed by a third pregnancy in which RAI was diagnosed prenatally. After the diagnosis, she did not return for a follow up. Although the most cases of atrial isomerism are sporadic, familial occurrence has also been reported [1720]. Kaasinen et al. [21] performed a genetic analysis on five children with RAI in the same family and confirmed that RAI can occur as a recessively inherited condition, caused by a mutation in the growth/differentiation factor 1 (GDF1) gene. Because we only performed routine karyotyping, we did not look at inheritance patterns. Little is known about the genetics of congenital heart defects and thus, further studies are needed to confirm the genes responsible for inherited atrial isomerism. Although unlikely, practitioners should offer a fetal echocardiography to mothers who previously conceived a fetus with atrial isomerism.

Prenatal ultrasonographic findings of one fetus with LAI in our study showed an interrupted IVC, suspected coarctation 


\section{Obstetrics \& Gynecology Science}

Vol. 57, No. 3, 2014

Table 3. Reported cases of atrial isomerism and postnatal outcomes

\begin{tabular}{|c|c|c|c|c|c|c|c|c|c|c|c|c|}
\hline \multirow[b]{2}{*}{ Study } & \multicolumn{6}{|c|}{ RAI } & \multicolumn{6}{|c|}{ LAI } \\
\hline & $\begin{array}{c}\text { Cases } \\
\text { (n) }\end{array}$ & $\begin{array}{c}\text { FU loss } \\
\text { (n) }\end{array}$ & $\begin{array}{c}\text { TOP } \\
\text { (n) }\end{array}$ & $\begin{array}{c}\text { FDIU } \\
\text { (n) }\end{array}$ & $\begin{array}{l}\text { LB } \\
\text { (n) }\end{array}$ & $\begin{array}{c}\text { Alive } \\
(\%)\end{array}$ & $\begin{array}{c}\text { Cases } \\
\text { (n) }\end{array}$ & $\begin{array}{c}\text { FU loss } \\
\text { (n) }\end{array}$ & $\begin{array}{l}\text { TOP } \\
\text { (n) }\end{array}$ & $\begin{array}{c}\text { FDIU } \\
(n)\end{array}$ & $\begin{array}{l}\text { LB } \\
\text { (n) }\end{array}$ & $\begin{array}{c}\text { Alive } \\
(\%)\end{array}$ \\
\hline Atkinson et al. (1998) [3] & 8 & 0 & 1 & 0 & 7 & $1(14)$ & 5 & 0 & 2 & 0 & 3 & $1(33)$ \\
\hline Lin et al. (2002) [4] & 25 & 10 & 6 & 0 & 9 & $4(44)$ & 4 & 2 & 1 & 0 & 1 & $1(100)$ \\
\hline Berg et al. (2003) [5] & 10 & 0 & 0 & 1 & 9 & $4(44)$ & 22 & 0 & 12 & 2 & 8 & $8(100)$ \\
\hline Lim et al. (2005) [7] & 31 & 0 & 14 & 0 & 17 & $2(12)$ & 52 & 0 & 20 & 2 & 30 & $22(73)$ \\
\hline Yan et al. (2008) [9] & 18 & 0 & 15 & 0 & 3 & $3(100)$ & 4 & 0 & 3 & 0 & 1 & $0(0)$ \\
\hline Present study & 37 & 3 & 9 & 0 & 25 & $15(60)$ & 34 & 1 & 2 & 0 & 31 & $28(90)$ \\
\hline
\end{tabular}

RAI, right atrial isomerism; LAI, left atrial isomerism; FU, follow up; TOP, termination of pregnancy; FDIU, fetal death in utero; LB, live birth.
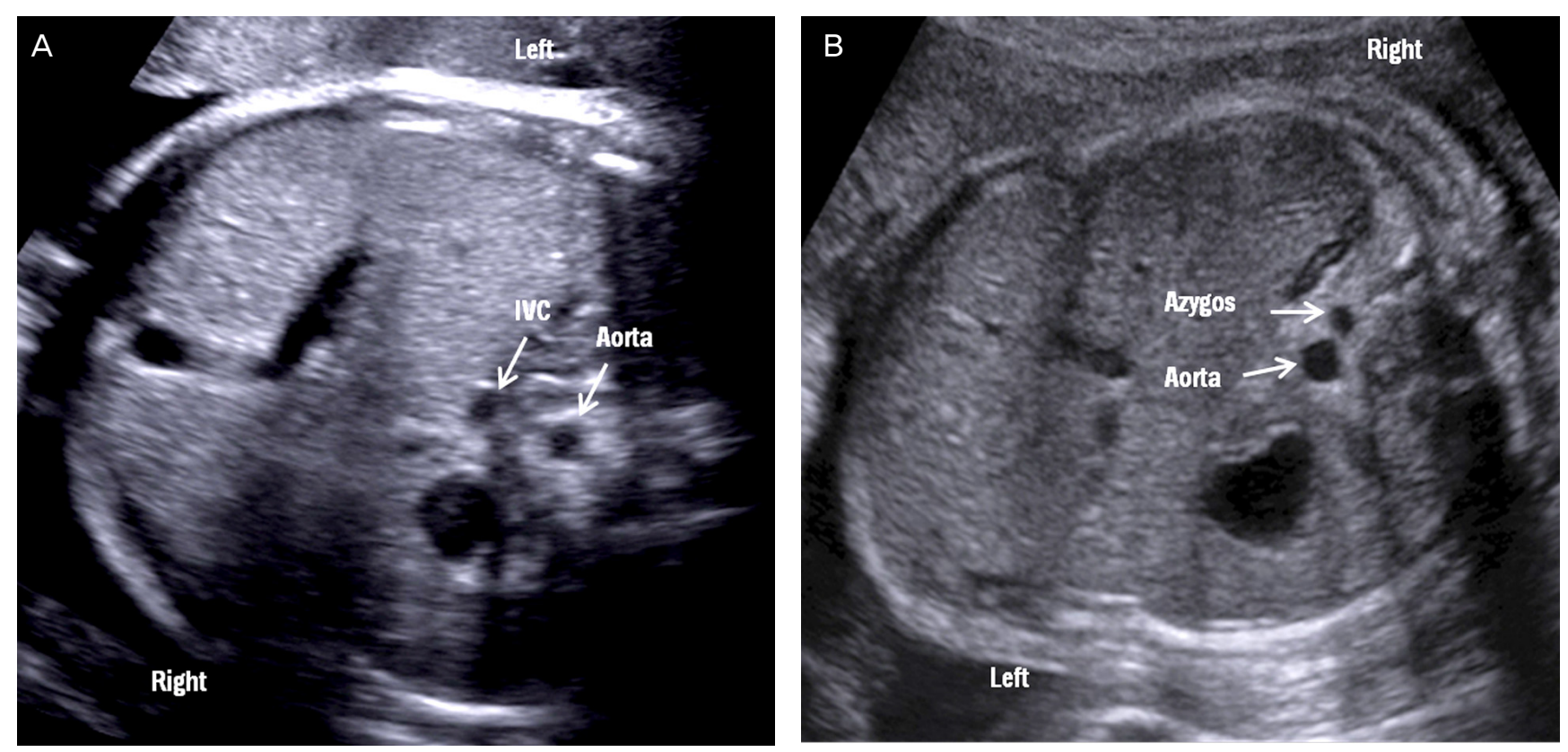

Fig. 2. Ultrasonographic diagnostic clues for both forms of atrial isomerism. (A) Right atrial isomerism with juxtaposition of the aorta and inferior vena cava (IVC) on the same side of the spine (arrows). Right-sided stomach and midline liver are also noted. (B) Left atrial isomerism with interruption of the IVC and azygos vein continuation, showng a 'double vessel' sign (arrows). Lee MY, et al. Obstet Gynecol Sci 2013;56:217-26, according to the Creative Commons Attribution License [27].

of aorta with polysplenia, and the postnatal evaluation only confirmed IVC interruption with a normal heart and spleen. Although the majority of cases with IVC interruption are associated with visceral heterotaxy, especially in LAI, it can occur as an isolated finding, which is very rare [22]. When IVC is absent by ultrasonography, examiners should keep in mind the possibility of associated cardiac anomalies, and if no abnormalities are detected, the prognosis is excellent unlike that associated with LAI [23].

Patients with RAI show a higher mortality rate because of more complex cardiac malformations and asplenia, which increases the risks of dying from sepsis $[24,25]$. We reviewed five literatures reporting significant numbers of prenatally diagnosed both atrial isomerism cases and their postnatal outcomes (Table 3). All but one demonstrated a lower survival rate for RAl than for LAI. Since the study by Yan et al. [9] reported only a very small number of survivors, it was not possible to conclude whether the differences in survival rates were statistically significant. Our study demonstrated better survival rates, with a greater number of live births than any other study, especially on RAl. The advances in surgical techniques along with a conservative management for complex 


\section{Obstetrics \& Gynecology Science}

Mi-Young Lee, et al. Prenatal diagnosis of atrial isomerism

heart disease have improved the long term prognosis. A recent series by Ota et al. [26] showed that five-year survival of patients with RAI to be $53.8 \%$ before 2003 and $81.7 \%$ after 2004. They concluded that the survival rate improved with the use of aggressive treatments for concomitant anomalies and refinement of surgical techniques.

Accurate prenatal diagnosis of both forms of atrial isomerism is important in order to counsel parents, plan a prompt cardiac surgery after birth, and treat the associated abnormalities. RAI should be suspected when complex heart disease, commonly associated with atrioventricular septal defect, juxtaposition of the aorta and IVC, and visceral abnormalities are present (Fig. 2A). LAl can be diagnosed even in a normal heart. The most consistent marker of LAI is an interrupted IVC with azygos vein continuation, which is diagnosed by the identification of the 'double vessel' sign on a transverse view of the upper abdomen with the absence of IVC in its usual location (Fig. 2B) [28].

Our study confirms the previous findings of fetal atrial isomerism except a much lower prevalence of atrioventricular septal defect and complete heart block in LAl. Although the postnatal outcomes for RAI were worse than those for LAl, successful postnatal surgery with active management improved the survival rate. Parents of fetuses diagnosed with atrial isomerism should be counseled carefully about the prognosis, encouraged to continue the pregnancy and provided with proper postnatal treatment.

\section{Conflict of interest}

No potential conflict of interest relevant to this article was reported.

\section{References}

1. Ho SY, Cook A, Anderson RH, Allan LD, Fagg N. Isomerism of the atrial appendages in the fetus. Pediatr Pathol 1991;11:589-608.

2. Phoon CK, Villegas MD, Ursell PC, Silverman NH. Left atrial isomerism detected in fetal life. Am J Cardiol 1996;77:1083-8.

3. Atkinson DE, Drant S. Diagnosis of heterotaxy syndrome by fetal echocardiography. Am J Cardiol 1998;82:1147-9.
4. Lin JH, Chang $\mathrm{Cl}$, Wang JK, Wu MH, Shyu MK, Lee CN, et al. Intrauterine diagnosis of heterotaxy syndrome. Am Heart J 2002;143:1002-8.

5. Berg C, Geipel A, Smrcek J, Krapp M, Germer U, Kohl T, et al. Prenatal diagnosis of cardiosplenic syndromes: a 10-year experience. Ultrasound Obstet Gynecol 2003;22:451-9.

6. Berg C, Geipel A, Kamil D, Knuppel M, Breuer J, Krapp $M$, et al. The syndrome of left isomerism: sonographic findings and outcome in prenatally diagnosed cases. J Ultrasound Med 2005;24:921-31.

7. Lim JS, McCrindle BW, Smallhorn JF, Golding F, Caldarone CA, Taketazu M, et al. Clinical features, management, and outcome of children with fetal and postnatal diagnoses of isomerism syndromes. Circulation 2005; 112:2454-61.

8. Taketazu M, Lougheed J, Yoo SJ, Lim JS, Hornberger LK. Spectrum of cardiovascular disease, accuracy of diagnosis, and outcome in fetal heterotaxy syndrome. Am J Cardiol 2006;97:720-4.

9. Yan YL, Tan KB, Yeo GS. Right atrial isomerism: preponderance in Asian fetuses. Using the stomachdistance ratio as a possible diagnostic tool for prediction of right atrial isomerism. Ann Acad Med Singapore 2008;37:906-12.

10. Pepes S, Zidere V, Allan LD. Prenatal diagnosis of left atrial isomerism. Heart 2009;95:1974-7.

11. Nakada K, Kawaguchi F, Wakisaka M, Nakada M, Enami T, Yamate N. Digestive tract disorders associated with asplenia/polysplenia syndrome. J Pediatr Surg 1997;32:91-4.

12. Ticho BS, Goldstein AM, Van Praagh R. Extracardiac anomalies in the heterotaxy syndromes with focus on anomalies of midline-associated structures. Am J Cardiol 2000;85:729-34.

13. Hsu JY, Chen SJ, Wang JK, Ni YH, Chang MH, Wu MH. Clinical implication of hiatal hernia in patients with right isomerism. Acta Paediatr 2005;94:1248-52.

14. Chang J, Brueckner M, Touloukian RJ. Intestinal rotation and fixation abnormalities in heterotaxia: early detection and management. J Pediatr Surg 1993;28:1281-4.

15. Vazquez J, Lopez Gutierrez JC, Gamez M, Lopez-Santamaria M, Murcia J, Larrauri J, et al. Biliary atresia and the polysplenia syndrome: its impact on final outcome. $J$ Pediatr Surg 1995;30:485-7.

16. Ruano R, Benachi A, Aubry MC, Bernard JP, Hameury F, 


\title{
Obstetrics \& Gynecology Science
}

\author{
Vol. 57, No. 3, 2014
}

Nihoul-Fekete C, et al. Prenatal sonographic diagnosis of congenital hiatal hernia. Prenat Diagn 2004;24:26-30.

17. Simpson J, Zellweger H. Familial occurrence of Ivemark syndrome with splenic hypoplasia and asplenia in sibs. J Med Genet 1973;10:303-4.

18. Rose V, Izukawa T, Moes CA. Syndromes of asplenia and polysplenia. A review of cardiac and non-cardiac malformations in 60 cases withspecial reference to diagnosis and prognosis. Br Heart J 1975;37:840-52.

19. Zlotogora J, Elian E. Asplenia and polysplenia syndromes with abnormalities of lateralisation in a sibship. J Med Genet 1981;18:301-2.

20. Eronen M, Kajantie E, Boldt T, Pitkanen O, Aittomaki K. Right atrial isomerism in four siblings. Pediatr Cardiol 2004;25:141-4.

21. Kaasinen E, Aittomaki K, Eronen M, Vahteristo P, Karhu A, Mecklin JP, et al. Recessively inherited right atrial isomerism caused by mutations in growth/differentiation factor 1 (GDF1). Hum Mol Genet 2010;19:2747-53.

22. Celentano C, Malinger G, Rotmensch S, Gerboni S, Wolman $Y$, Glezerman M. Prenatal diagnosis of interrupted inferior vena cava as an isolated finding: a benign vascular malformation. Ultrasound Obstet Gynecol 1999;14:215-8.

23. Bronshtein M, Khatib N, Blumenfeld Z. Prenatal diagnosis and outcome of isolated interrupted inferior vena cava. Am J Obstet Gynecol 2010;202:398.e1-4.

24. Freedom RM, Jaeggi ET, Lim JS, Anderson RH. Hearts with isomerism of the right atrial appendages: one of the worst forms of disease in 2005. Cardiol Young 2005;15:554-67.

25. Waldman JD, Rosenthal A, Smith AL, Shurin S, Nadas AS. Sepsis and congenital asplenia. J Pediatr 1977;90:555-9.

26. Ota N, Fujimoto Y, Murata M, Tosaka Y, Ide Y, Tachi M, et al. Improving outcomes of the surgical management of right atrial isomerism. Ann Thorac Surg 2012;93:832-8.

27. Lee MY, Won HS. Technique of fetal echocardiography. Obstet Gynecol Sci 2013;56:217-26.

28. Sheley RC, Nyberg DA, Kapur R. Azygous continuation of the interrupted inferior vena cava: a clue to prenatal diagnosis of the cardiosplenic syndromes. J Ultrasound Med 1995;14:381-7. 